\title{
Selbsttötung als Menschenrecht - ethische Überlegungen zu einem gesellschaftlichen Klimawandel
}

\author{
Werte und Normen prägen das Klima einer Gesellschaft und die Art und Weise, \\ wie Menschen zusammenleben. Ein Wertewandel führt denn auch zu einem \\ gesellschaftlichen Klimawandel. Der folgende Artikel setzt sich mit der ver- \\ änderten gesellschaftlichen Haltung gegenüber dem Suizid auseinander, die \\ sich jüngst in einem Bundesgerichtsurteil zeigt, und hinterfragt die gesell- \\ schaftlichen Konsequenzen dieses Wertewandels.
}

Ruth Baumann-Hölzle

Korrespondenz:

Dr. theol. Ruth Baumann-Hölzle

Institut Dialog Ethik

Sonneggstrasse 88

CH-8006 Zürich

rbaumann@dialog-ethik.ch

\section{Gesellschaftlicher Klimawandel}

So wie sich das Klima in der Natur ändert, wandelt sich auch das gesellschaftliche Klima des Zusammenlebens spürbar. Werte und Normen erhalten eine neue Bedeutung, und Gesetze werden verändert oder neue geschaffen. Gesetze vollziehen den Wertewandel oft affirmativ passiv nach, können ihn aber auch fördern oder bremsen. So haben etwa die Gleichstellungsgesetze die Besserstellung der Frauen, der behinderten Menschen und der gleichgeschlechtlichen Paare vorangetrieben und das moralische Gesellschaftsklima positiv beeinflusst. Im Gegensatz dazu haben die Gesetzesänderungen vor dem Zweiten Weltkrieg die Entwertung der Juden und behinderten Menschen innerhalb der Gesellschaft massiv gefördert und die darauf- folgenden menschenverachtenden Handlungen vorbereitet. Neue Gesetze sind deshalb stets auf ihre moralischen Voraussetzungen und auf ihre Implikationen für das Klima des Zusammenlebens hin ethisch zu reflektieren.

Vor diesem Hintergrund hinterfragt der vorliegende Artikel das Bundesgerichtsurteil vom 3. November 2006 2A.48/2006/2A.66/2006, das die Möglichkeit der Selbsttötung neu als ein Menschenrecht formuliert: «Zum Selbstbestimmungsrecht im Sinne von Artikel 8 EMRK (sc. Europäische Menschenrechtskonvention) gehört auch das Recht, über Art und Zeitpunkt der Beendigung des eigenen Lebens zu entscheiden; dies zumindest, soweit der Betroffene in der Lage ist, seinen entsprechenden Willen frei zu bilden und danach zu handeln» (6.1). Demgegenüber lehnt das Bundesgericht in diesem Urteil ein Recht auf Beihilfe zum Suizid explizit ab.

\section{Le suicide, un droit humain: réflexions éthiques concernant} l'évolution des mentalités

Les valeurs et normes marquent une société et la manière avec laquelle les personnes vivent ensemble. Lorsque les valeurs changent, les mentalités changent aussi. Dans I'article ci-après, I'auteur examine cette évolution face au suicide, telle qu'elle ressort d'un arrêt récent du Tribunal fédéral et s'interroge sur les conséquences de ce changement de valeurs pour la société.

\section{Moralisches Klima und Rechtsetzung}

Ethik und Recht haben eines gemeinsam: Sie kommen in der Regel zu spät, nämlich dann, wenn die Selbstverständlichkeit des Handelns bereits zerbrochen ist. Beide suchen sie nach Antworten auf die Frage: Was sollen wir tun? Die Ethik eilt dabei dem Recht voraus, denn noch bevor der Gesetzgeber sich in der Frage nach dem erlaubten Tun festgelegt hat, haben die betroffenen Menschen bereits eine persönliche Entscheidung getroffen und vielleicht gar schon gehandelt. Entscheide erzeugen in einer Gesellschaft ein bestimmtes moralisches Klima, welches sich auf den folgenden Gesetzgebungsprozess auswirkt und die Gesetzesinhalte prägt. Umgekehrt beeinflusst auch die Rechtsetzung das Klima des Zusammenlebens.

Beim gesellschaftlichen Wertewandel verändert sich das Gewicht bestimmter Werte und 
Normen. Wie der Klimawandel der Natur vollzieht sich auch der gesellschaftliche Klimawandel zuerst unbemerkt und träge in kleinen Schritten. Die grundsätzliche Bedeutung der einzelnen Schritte für das gesellschaftliche Klima wird oft erst im Rückblick sichtbar, wenn das ganze Ausmass der Werteveränderungen erkennbar wird. Der Gesetzgebungsprozess legitimiert vorerst diese kleinen Schritte mit neuen Gesetzen, noch bevor der Wertewandel in seiner Bedeutung für das Zusammenleben der Menschen wahrgenommen worden ist. Im Rahmen des Gesetzgebungsprozesses beschränkt sich die öffentliche Debatte denn auch meist auf die Auseinandersetzung mit den kleinen Schritten. Sind erste Schritte einmal gemacht, geht es häufig sehr rasch. Das gesellschaftliche Klima kippt, ohne dass der Wertewandel noch gestoppt werden könnte. Auch Gesetze werden dann sehr schnell geändert.

Das liberale Staatsrecht, das Völkerrecht und die Menschenrechte basieren auf dem unverlierbaren und wesenhaften Würde- und Autonomieanspruch jedes Menschen. Die Menschenwürde begründet den Anspruch des Menschen auf Schutz und Respekt gegenüber seiner Integrität. Jeder Mensch hat deshalb in jeder Hinsicht ein fundamentales Abwehrrecht und darf nicht von fremden Interessen vereinnahmt werden. Jeder Mensch hat Anspruch auf Respekt gegenüber seiner physischen und psychischen Integrität. Für lebensrettende Eingriffe und Therapien braucht es deshalb die Einwilligung des betroffenen Menschen. Insofern toleriert ein liberaler Staat selbstschädigendes Verhalten. Auch Menschen, die in einem urteilsfähigen Zustand einen Suizid begehen, haben das Recht, lebenserhaltende Massnahmen zu verweigern, seien sie nun psychisch krank oder nicht. Dieses Abwehrrecht ist nur im Falle einer Fremdgefährdung verhandelbar, sonst gilt es absolut.

So gesehen sind Rechte im pluralistischen demokratischen Staat Grenzziehungen persönlicher Handlungsspielräume, innerhalb deren jede Person nach ihrer eigenen Moral leben kann, solange sie nicht die Freiheitsgrenze anderer Menschen verletzt. In einem demokratisch verfassten, liberalen Staat wird bei der Frage nach dem legitimen Tun der kleinste gemeinsame moralische Nenner gesucht, um den Bürgerinnen und Bürgern möglichst grosse Handlungsspielräume zu ermöglichen. Es steht den einzelnen jedoch frei, für das eigene Leben engere Handlungsgrenzen zu ziehen. Jedes Recht ist damit mit einem moralischen Freiheitsanspruch hinterlegt. Umgekehrt muss aber nicht jeder Freiheitsanspruch mit einem Recht korrespon- dieren, denn innerhalb des durch Rechte abgegrenzten Handlungsspielraums besteht die Freiheit, mit seinem Leben tun und lassen $\mathrm{zu}$ können, was man will. Der Staat aber hat kein Recht, in diesen Privatraum einzugreifen, solange der Mensch urteilsfähig ist und keine Fremdgefährdung besteht.

Rechte setzen aber nicht nur Grenzen, sondern üben auch eine moralische Wirkung aus. Als moralisches Minimum sind sie auch moralischer Appell: Das, was rechtlich erlaubt ist, sei moralisch nicht nur vertretbar, sondern auch richtungsweisend. In dieser befürwortenden Art und Weise werden bestimmte Handlungen von der Gesellschaft nicht nur toleriert, sondern als gleichberechtigte Handlungsoptionen in den Handlungskanon einer Gesellschaft aufgenommen.

\section{Bisherige Rechtslage in der Schweiz}

In der Schweiz herrscht in bezug auf den Suizid und die Beihilfe zum Suizid im Vergleich mit dem benachbarten Ausland eine sehr liberale Praxis. Nach Artikel 115 StGB ist die Beihilfe zur Selbsttötung straflos, wenn sie nicht aus selbstsüchtigen Motiven geschieht. Der Staat wacht darüber, dass der Entscheid zum Suizid oder die Beihilfe zum Suizid absolut freiwillig und nicht aufgrund von Fremdinteressen gefällt wird. Mit dieser Gesetzgebung wird in der Schweiz der Schutz der Willensfreiheit höher gewertet als der Lebensschutz. Die Möglichkeit zum Suizid und die Beihilfe werden rechtlich auf der individualethischen Ebene als Ultima-ratioHandlungen in einer ausweglosen Situation als Gewissensentscheide zweier Menschen toleriert, die sich nahestehen, erlaubt und nicht als Tötungsdelikte oder Unterlassene Hilfeleistung geahndet. Bei der Einführung dieser gesetzlichen Regelung war die Möglichkeit von Sterbehilfeorganisationen nicht im Blick, welche die Beihilfe zum Suizid ausserhalb persönlicher Beziehungen und Gewissensentscheide durchführen.

Dieser individualethischen Freiheit zur Selbsttötung und zu deren Beihilfe steht sozialethisch die Verpflichtung des Staates zum allgemeinen Lebensschutz gegenüber. Trotz der individuellen Freiheit zum Suizid hat deshalb der Staat die Möglichkeit, bei Selbst- oder Fremdgefährdung Menschen mit einem sogenannten «Fürsorgerischen Freiheitsentzug (FFE)» zu schützen. Der FFE kann aber nur so lange aufrechterhalten werden, bis geklärt ist, aus welchen Gründen ein Mensch sich töten will. Nur wenn eine psychische Erkrankung vorliegt oder der Entscheid zum Suizid aufgrund 
äusseren Drucks entstanden ist, darf ein Mensch vor sich selbst geschützt werden. Gegen seinen Willen hingegen darf niemand am Leben erhalten werden. Entsprechend gelten juristisch alle medizinischen und pflegerischen Massnahmen als Körperverletzung, die durch die Einwilligung des Patienten, d.h. seine informierte Zustimmung, ihre Widerrechtlichkeit verlieren. Auch bei Heilbehandlungen bleibt juristisch der Tatbestand der Körperverletzung bestehen.

Der Schutz der Willens- und Gewissensfreiheit ist eine Kernaufgabe eines demokratisch verfassten Staates. Die Trennung zwischen öffentlichem und privatem Raum ist im liberalen Staat zentral. Persönliche Freiheiten dürfen nur dann beschnitten werden, wenn daraus Gefährdungen für Dritte erwachsen. Die sozialethische Schutzverpflichtung der Lebenserhaltung steht dann über dem individuellen Freiheitsanspruch. Aus diesem Grund wird vermieden, die Option der Tötung in den sozialethischen Kontext einfliessen zu lassen. Vor diesem Hintergrund wurden bis anhin der Suizid und die Beilhilfe zum Suizid als Freiheit toleriert, die jedoch nicht als Recht eingefordert werden konnten. Darin widerspiegelt sich der rechtliche verbriefte Autonomieanspruch des einzelnen Menschen, wonach er jegliche Handlung als eine Integritätsverletzung verweigern, jedoch nicht alle Handlungen verlangen kann.

\section{Der Suizid als Menschenrecht}

Der Bundesgerichtsentscheid zum Suizid und zur Suizidbeihilfe orientiert sich denn bei seiner Argumentationsführung auch an dieser Trennung von öffentlichem und privatem Raum, indem das Gericht auf der einen Seite klar die Suizidbeihilfe als staatliche Aufgabe ablehnt, auf der anderen Seite den Suizid der Privatsphäre zuordnet und dort als Persönlichkeitsrecht formuliert. In gleicher Art und Weise ermöglichen auch die Richtlinien der SAMW bezüglich der Entscheide am Lebensende dem einzelnen Arzt die Suizidbeihilfe bei Personen am Lebensende als persönlichen, privaten Gewissensentscheid, lehnen jedoch die Suizidbeihilfe als zur ärztlichen Tätigkeit gehörende und damit als öffentlich soziale Aufgabe ab.

Bezüglich der Trennung der privaten und der öffentlichen Sphäre ist nun aber das Bundesgericht nicht stringent. Es stellt nämlich die Möglichkeit zum Suizid nicht mehr nur als eine Freiheitsoption dar, die indirekt aus dem Abwehrrecht des Individuums gegenüber lebenserhaltenden Massnahmen entsteht, sondern formuliert den Suizid als «Recht auf den eigenen Tod» (6.2.1), das es direkt aus der EMRK
Art. 8 Ziff. 1 (Gebot der Achtung der Privatsphäre ableitet: «Das Recht auf Achtung des Privatlebens gemäss Art. 8 Ziff. 1 EMRK sichert dem Individuum einen Freiraum $\mathrm{zu}$, in dem es seine Persönlichkeit entwickeln und verwirklichen kann; es soll unter Ausschluss des Staates im Rahmen der privaten Sphäre über die eigene Person und die Gestaltung des Lebens verfügen können [...].» Zum Selbstbestimmungsrecht im Sinne von Art. 8 EMRK gehört auch das Recht, über Art und Zeitpunkt der Beendigung des eigenen Lebens zu entscheiden; dies zumindest, soweit der Betroffene in der Lage ist, seinen entsprechenden Willen frei zu bilden und danach zu handeln (6.1). Mit diesem Bezug auf die Europäische Menschenrechtskonvention hat ein Urteilsfähiger nicht nur ein Menschenrecht, sein Leben selbst zu gestalten, sondern auch das Menschenrecht auf Selbsttötung. Folglich spricht das Bundesgericht faktisch dem einzelnen nicht nur ein «Recht auf den eigenen Tod» zu, sondern ein «Recht auf Selbsttötung», d.h. mit anderen Worten, der Suizid wird zum Menschenrecht, das jedoch nicht vom Staat eingefordert werden kann.

Das «Recht auf den eigenen Tod» erhält so eine neue Bedeutung. Solange die medizinischen Möglichkeiten der Lebenserhaltung beschränkt waren, war der Tod als das schlechthin Unverfügbare dem Handeln des Menschen entzogen. In diesem Kontext bedeutete das «Recht auf den eigenen Tod» das Recht, nicht getötet zu werden. Vor dem Hintergrund des neuen Könnens der Medizin, welches Leben unter Umständen auch über das erträgliche Mass eines Menschen hinaus verlängern kann, wurde dieses Recht zur Kampfformel gegen medizinische Übertherapie. Das «Recht auf den eigenen Tod» bedeutete in diesem Kontext das Recht, lebenserhaltende Massnahmen zu verweigern. Als Abwehrformel sollte das «Recht auf den eigenen Tod» die Handlungsmacht des Menschen beschränken, und der Tod sollte als das vom Menschen Erlittene und Unverfügbare, das Fremde, wie Christoph Rehmann-Sutter in seinem Artikel schreibt, als Vorgegebenheit respektiert werden. Dieses Abwehrrecht gegenüber lebenserhaltenden Massnahmen erweitert nun das Bundesgericht $\mathrm{zu}$ einem Anspruchsrecht auf Selbsttötung: Das Menschenrecht auf den eigenen Tod schliesst neu auch das Menschenrecht auf Selbsttötung ein.

Indem das Bundesgericht die Freiheit zum Suizid als Recht auf Suizid legitimiert, ist die Selbsttötung keine reine private Angelegenheit mehr, sondern auch eine staatlich legitimierte. Das Bundesgericht hat diese Freiheit zur Selbst- 
tötung als Menschenrecht aus dem Graubereich des rechtsfreien Raumes herausgenommen. Mit diesem Recht wird die Möglichkeit des Suizids $\mathrm{zu}$ einer vom Staat rechtlich und moralisch vertretbaren Handlung. Mit dem Suizidrecht ist die Option der Tötung in der Schweiz in den sozialen Organismus der Gesellschaft aufgenommen worden. Dies hat weitreichende Konsequenzen für das moralische Klima der Gesellschaft.

In letzter Konsequenz verzichtet in diesem Bundesgerichtsurteil der Staat auf eine moralische Bewertung der Selbsttötung eines urteilsfähigen Menschen: $\mathrm{Ob}$ der Mensch sein Leben erhält oder ob er sich tötet, ist für den Staat irrelevant. Er hat nur sicherzustellen, dass der Entscheid zur Selbsttötung ohne Druck von aussen getroffen wird. Der Staat beschränkt sich damit auf einen Minimalstandard. Christian Schwarzenegger schreibt hierzu: «Das verfassungsrechtlich geschützte Recht auf Leben dürfte den Staat im vorliegenden Zusammenhang als Minimalvorgabe vorab dazu verpflichten, durch ein geeignetes Verfahren sicherzustellen, dass ein allfälliger Entscheid über die Beendigung des Lebens tatsächlich dem freien Willen des Betroffenen entspricht» (Erw. 6.2.1) (Hervorhebung im Text durch den Autor selbst).

In die gleiche Richtung zielt auch die Motion von Hansruedi Stadler, die im Juni 2007 vom Ständerat mit $17 \mathrm{zu} 9$ Stimmen überwiesen worden ist. Die Motion fordert eine Aufsicht über Suizidhilfeorganisationen, die sicherstellen soll, dass der Entscheid ohne Druck von aussen und wohlüberlegt gefällt worden ist. Suizidhilfeorganisationen begrüssen dieses Vorgehen, denn damit erhält ihre Tätigkeit die moralische Legitimation durch die Gesellschaft. Suizidbeihilfe ist damit definitiv keine Ultima-ratio-Handlung mehr, sondern eine vom Staat sanktionierte Tätigkeit. Die Beihilfe zur Selbsttötung soll denn auch standardisiert und professionalisiert werden. Mit einer solchen Aufsicht über Suizidhilfeorganisationen geht auch die Suizidbeihilfe aus dem privaten Freiraum in den öffentlichen Raum über.

Der säkulare Staat enthält sich mit diesem Recht auf Selbsttötung jeglicher moralischer Stellungnahme gegenüber Leben und Tod eines Menschen. Weder wird sein Leben gewürdigt noch sein Tod bedauert. Christian Schwarzenegger begründet dieses Vorgehen damit, dass der Staat damit keine Privatmoral zur Staatsmoral erheben würde. Es stellt sich aber die Frage, ob einer humanen Gesellschaft der Tod eines Menschen gleichgültig sein kann. Nach Christoph Rehmann-Sutter würden mit dem Recht auf den eigenen Tod dagegen die auch innerhalb der Freiheit zum Suizid noch bestehenden fürsorgenden Verantwortungen anderer, mit denen der Sterbewillige in Beziehung steht, von vornherein übertrumpfen. Eine solch fürsorgende Verantwortung ist aber nur noch hinsichtlich der Sicherstellung der Entscheidungsfreiheit des Suizidanten gegeben. Angesichts des Rechts auf Selbsttötung fällt es schwer, staatliche Präventionsmassnahmen gegen den Suizid oder auch selbstschädigendes Verhalten zu begründen. Solange sie von urteilsfähigen Menschen ausgeführt werden, trägt der Staat keine Fürsorgeverantwortung mehr.

Das Bundesgericht hat mit diesem Entscheid einen Wertewandel legitimiert: Selbstschädigendes Verhalten wird so vom säkularen Staat nicht wie bis anhin nur toleriert, sondern als Recht legitimiert und moralisch sogar befürwortet. Sobald Handlungen befürwortet werden, entsteht auch eine moralische Verpflichtung, Menschen $\mathrm{zu}$ solchen Handlungen von der Gesellschaft $\mathrm{zu}$ verhelfen. Das Bundesgericht hat sich zwar explizit dagegen ausgesprochen, Suizidbeihilfe vom Staat einfordern zu können, aber mit der Bejahung des Suizids als eines Menschenrechts geht der moralische Appell einher, einem suizidwilligen Menschen zur Selbsttötung zu verhelfen. Damit muss sich moralisch nicht mehr derjenige rechtfertigen, der die Suizidbeihilfe durchführt, sondern derjenige, der sie verweigert. Dies ist besonders für die Ärzteschaft bedeutsam. Denn nach Christian Schwarzenegger rückt mit der Rezeptpflicht für Natrium-Pentobarbital das Bundesgericht die Schweizer Praxis näher an ein System des ärztlich assistierten Suizids heran, weil in jedem Fall eine medizinische Untersuchung erfolgen muss. Seitdem das Bundesgerichtsurteil bekanntgeworden ist, haben denn auch Vertreter der Ärzteschaft gegen diesen moralischen Anspruch auf Suizidbeihilfe Einspruch erhoben, so auch René Raggenbass und Hanspeter Kuhn in ihrem Artikel «Kein Menschenrecht auf Suizidbeilfe» und Claude Regamey und Michelle Salathé mit ihren Ausführungen «Ärztinnen und Ärzte sind nicht Experten für den freiwilligen Tod». Stellung genommen hat auch die Schweizerische Gesellschaft für Forensische Psychiatrie SGFP.

Dies sind Hinweise dafür, dass das gesellschaftliche Klima bereits weiter fortgeschritten ist und sich die moralische Verpflichtung der Gesellschaft abzeichnet, Suizidbeihilfe leisten zu müssen. Es überrascht denn auch nicht, dass eine Presseagentur nach Bekanntwerden des Bundesgerichtsurteils fälschlicherweise die Meldung verbreitete, in der Schweiz sei die 
Suizidbeihilfe als Menschenrecht für psychisch Kranke formuliert worden, was wiederum weitere Falschmeldungen nach sich zog.

\section{Der Suizid als finales Lebensevent}

Dem Suizid gegenüber zeichnet sich gesellschaftlich grundsätzlich eine Haltungsänderung ab: Der Suizid wird zum finalen Lebensevent des autonomen Individuums. So zelebriert, übt der Suizid einen moralischen Appell aus, es dem Suizidanten in ähnlichen Lebenssituationen gleichzutun. Dieser Appell kann leicht zum sozialen Zwang werden.

\section{Relativierung der Menschenwürde hilfsbedürftiger Menschen}

Während auf der einen Seite der Suizid als Menschenrecht aufgewertet und zum Event stilisiert wird, wird gleichzeitig die Menschenwürde für bestimmte Menschengruppen relativiert und von ihrem Funktionieren abhängig gemacht. So wird ohne Scheu öffentlich die Frage aufgeworfen, ob zum Bespiel demente oder inkontinente Menschen noch Menschenwürde besitzen würden. Die Menschenrechte, wie sie in der Deklaration von Helsinki für jeden Menschen festgeschrieben worden sind, gründen auf einem Verständnis von Menschenwürde, das jedem Menschen unabhängig von Eigenschaften und Fähigkeiten Würde zuspricht. Wer die Menschenwürde relativiert, relativiert deshalb auch die Menschenrechte. Die moralische Vertretbarkeit des Suizids wird öfters mit dem Verlust der Menschenwürde begründet. Wird aber bestimmten Menschen die Würde abgesprochen, wird ihnen im Zuge dieses Denkens der Suizid nahegelegt. Hinter solchen Überlegungen stehen auch finanzielle Interessen.

\section{Suizid als sozialer Zwang}

Im Kontext von Mittelknappheit bei den Sozialwerken und angesichts der veränderten Bevölkerungspyramide kann ein sozialer Druck auf Schwache entstehen, dem Staat nicht zur Last zu fallen. Dieser Druck wird von betroffenen Menschen verinnerlicht und kann dann u.a. als Suizidwunsch in Erscheinung treten. Denn der Suizid ist effizient und kostensparend. So schreibt der Jurist F. Petermann in seinem Aufsatz zum Entwurf eines Gesetzes zur Suizidprävention, dass es angesichts explosionsartig steigender Gesundheitskosten angezeigt sei, «durch eine sinnvolle Legiferierung in diesem Bereich Einsparungen für das Gesundheitswesen vorzunehmen».

So hat Ruth Rieser, eine Frau, die mit einer Tetraplegie über fünfzig Jahre im Stadtspital
Triemli gelebt hatte und am 27. Mai 2007 verstorben ist, diesen Wandel erlebt. Früher hätten sie die Leute gefragt, was sie für sie tun könnten, heute werde sie gefragt, warum sie noch leben wolle: «Jemand fragte mich, als ich mich in einer schwierigen Lage befand: Weshalb kämpfst du eigentlich immer wieder für dieses Leben? Gib doch endlich auf, es lohnt sich schon lange nicht mehr.» Darauf antwortete sie: «Wer wollte dieses Sich-lohnen entscheiden?» Ich sagte: «Ich kämpfe nicht, ich lebe alles durch, was auf mich zukommt, das ganz Gute, aber auch das Schwierige. Soll ich vielleicht einfach aufgeben und das Leben womöglich wegschmeissen? Das Leben ist einfach da bis zum letzten Herzschlag. Für mich ist es fein und zart und sehr verletzlich, aber auch einfach eine Wucht und ein ganz grosses Geheimnis.» Ruth Rieser konnte ihr Leben nur deshalb so annehmen, weil ihr Umfeld sie optimal unterstützte: Sie malte, publizierte und arbeitete. Diese Solidarität wird zunehmend in Frage gestellt. Am Ende dieser Entwicklung steht die «Tötung aus Mitleid».

Und so verwischt sich heute bereits die Grenze zwischen Suizidbeihilfe und der in allen Fällen verbotenen aktiven Sterbehilfe zunehmend. Diesen menschenverachtenden Tendenzen ist von Anfang an zu widerstehen. Ich plädiere statt dessen dafür, die Selbsttötung weiterhin als tragische Handlung zu verstehen, die $\mathrm{zu}$ bedauern ist, auch wenn sie als persönlicher Freiheitsentscheid respektiert werden muss.

\section{Selbsttötung als tragische Handlung}

Die Selbsttötung ist im eigentlichen Sinne eine tragische Handlung, denn der leidende Mensch versucht das Leiden autonom loszuwerden, indem er sich selbst als autonome Person auslöscht. Sie ist eine Handlungsmöglichkeit des Menschen aufgrund eines Gewissensentscheides, angesichts deren die anderen Menschen nur schweigen können. Der Anspruch auf Gewissensfreiheit ist gesetzlich im Sinne eines Abwehrrechtes geschützt. Dieses Abwehrrecht gegenüber lebenserhaltenden Massnahmen eines suizidwilligen, urteilsfähigen Menschen impliziert aber weder einen moralischen Anspruch auf Suizid noch dessen moralische Vertretbarkeit. Der Suizidwillige trägt als urteilsfähige Person eine Verantwortung für sein Tun gegenüber dem eigenen Leben, aber auch den Angehörigen und der Gesellschaft gegenüber. Er hat sowohl das Leiden der ihm nahestehenden Menschen als auch die möglichen gesellschaftlichen Konsequenzen, die mit seiner Selbsttötung verbunden sind, mitzuverantworten. 
Andere Menschen können den Toten nur bedauern, beweinen und beklagen.

Indem die Rechtsetzung vor diesem Bundesgerichtsurteil die Beihilfe zum Suizid als eine Entscheidung aus Gewissensnot straffrei tolerierte, jedoch nicht als Menschenrecht legitimierte, wurde einerseits das Abwehrrecht des suizidwilligen Menschen gegenüber lebenserhaltenden Massnahmen respektiert und andererseits die Möglichkeit des Suizids nicht als rechtlich und moralisch vertretbar bewertet. Mit seinem Urteil hat das Bundesgericht für das gesellschaftliche Klima bei der gesellschaftlichen Haltung gegenüber dem Suizid ein widersprüchliches Zeichen gesetzt: Es unterstützt mit der Beurteilung des Suizids als Menschenrecht den gesellschaftlichen Klimawandel. Andererseits wirkt es diesem gegenüber auch bremsend, indem es den Anspruch auf Suizidbeihilfe abgelehnt hat.

Ein Suizid soll nicht zu einem moralischen Appell zur Nachahmung hochstilisiert werden können. Vielmehr soll er moralischer Appell an die Gesellschaft sein, möglichst gute Lebensund Sterbebedingungen für alle zu schaffen!

\section{Literatur}

- Bundesgericht 2A.48/2006; Ziffern in Klammern beziehen sich auf die Abschnittszählung.

- Petermann Frank T. Der Entwurf eines Gesetzes zur Suizid-Prävention. AJP/PJA. 2004;9:1111-38.

- Raggenbass R, Kuhn HP. Kein Menschenrecht auf ärztliche Suizidhilfe. Schweiz Ärztezeitung. 2007;88(11):455-6.

- Regamey C, Salathé M. Ärztinnen und Ärzte sind nicht Experten für den freiwilligen Tod. Schweiz Ärztezeitung. 2007;88(24):1051-2.

- Rehmann-Sutter C. Was bedeutet das «Recht auf den eigenen Tod»? Schweiz Ärztezeitung. 2007;88:(25):1109-12.

- Rieser R. «Ich liebe mein Leben trotz allem» aus dem Nachlass anlässlich ihrer Beerdigung vom 5. Juni 2007.

- Schwarzenegger C. Das Mittel zur Suizidbeihilfe und das Recht auf den eigenen Tod. Jusletter. 19. März 2007.

- Schweizerische Akademie der Medizinischen Wissenschaften. Medizinisch-ethische Richtlinien der SAMW. Betreuung von Patientinnen und Patienten am Lebensende. 2004.

- Kiesewetter M. Bundesgerichtsurteil zum assistierten Suizid von Psychischkranken (Verschreibungspflicht von Natrium-Pentobarbital). Schweiz Ärztezeitung. 2007;88(27/28):1195-7.

- Vonarburg V. Sterbehelfer sollen unter Bundesaufsicht kommen. Tages-Anzeiger. 22. Juni 2007:3. 